\title{
Chicago 4.0, dispepsia funcional en niños, fibra en trastornos funcionales intestinales, y genética y genómica en SII
}

Chicago 4.0, functional dyspepsia in children, fiber in functional intestinal disorders, and genetics and genomics in IBS

Max J. Schmulson ${ }^{1 *}$ y Miguel Saps ${ }^{2}$

${ }^{1}$ Laboratorio de Hígado, Páncreas y Motilidad (HIPAM)-Unidad de Investigación en Medicina Experimental, Facultad de Medicina-Universidad Nacional Autónoma de México (UNAM), Ciudad de México, México; ${ }^{2}$ Chief of Division of Pediatric Gastroenterology, Hepatology and Nutrition, University of MiamiMiller School of Medicine, Miami, Florida

En el tercero y último número del 2021 de NeuroGastroLATAM Reviews se publican cuatro artículos que tratan la publicación de la clasificación de Chicago (CCv4.0) en español, la dispepsia funcional en pediatría, el uso de fibra en los trastornos intestinales de la interacción intestino-cerebro (TIIC) y un primer en genética y genómica para el neurogastroenterólogo utilizando como ejemplo de estos conceptos los estudios realizados en síndrome de intestino irritable (SII) en este campo.

El primer artículo es la traducción autorizada del artículo original de la cuarta versión de la CCv4.0 para los trastornos motores del esófago, que fue publicada en Neurogastroenterology and Motility ${ }^{1}$, pero que consideramos importante publicar en español para nuestros lectores. Con el avance de la tecnología y en la actualidad casi la universalización de la

Correspondence to:

*Max Schmulson-Wasserman

E-mail:maxjulio@prodigy.net.mx manometría de alta resolución, ha sido necesario llevar a cabo este trabajo en progreso denominado la CCv4.0, que es la clasificación de los trastornos motores utilizando las métricas derivadas de esta metodología ${ }^{1}$. Esta nueva versión de la clasificación de Chicago es producto del trabajo de un diverso grupo de expertos de las sociedades y asociaciones de neurogastroenterología y motilidad del mundo, incluyendo a los doctores Albis Hani de Ardila de Colombia, Claudia Defilipi y Daniel Cisternas de Chile, y Enrique Coss-Adame de México, como representantes de la Sociedad Latinoamericana de Neurogastroenterología, y por supuesto coautores de la publicación original. La CCv4.0 recomienda un protocolo más estricto para la realización de los estudios, incluyendo valoración en posición supina y erguida, así como la realización de pruebas provocativas para determinar la presencia 
de reserva esofágica. Se modifican las métricas para valorar la unión esofagogástrica; y desde el punto de vista de los diagnósticos de los trastornos motores esofágicos, se refina la definición de la obstrucción de la unión esofagogástrica, así como de la motilidad esofágica inefectiva, que engloba ahora a la motilidad fragmentada ${ }^{2}$. La CCv4.0 ya no separa a los trastornos esofágicos como mayores o menores, sino que los separa en trastornos de la unión esofagogástrica y trastornos de la peristalsis. Además, se recomienda el uso de los estudios baritados para apoyar el diagnóstico o al menos realizar un diagnóstico previo mediante la radiología, para minimizar las ambigüedades de las clasificaciones previas ${ }^{2}$. Otro aspecto importante es que los diagnósticos se clasifican en conclusivos y no conclusivos. Es así como en acalasia, la CCv4.0 indica que debe haber un $100 \%$ de aperistalsis o estaríamos ante un diagnóstico no conclusivo de acalasia $^{3}$. Y en cuanto a la obstrucción del flujo de la unión esofagogástrica, los trastornos espásticos y el esófago hipercontráctil no tendrían significancia sin la presencia de pruebas de apoyo y manifestaciones clínicas ${ }^{3,4}$. Por supuesto, la clasificación no es completa y aún tiene limitantes, incluso ante hallazgos de alteraciones que no pueden ser clasificadas en las actualmente aceptadas, principalmente lo que antes denominábamos trastornos funcionales inespecíficos o los trastornos espásticos del esófago, pero como decíamos, este es un trabajo en progreso y futuras versiones deberán refinar aún más estos diagnósticos, con base en los hallazgos de la investigación. El grupo de Chicago 4.0 ha publicado además una serie de revisiones técnicas sobre los diversos trastornos específicos de la CCv4.0. El propósito de NeuroGastroLATAM Reviews es que los representantes de la Sociedad
Latinoamericana de Neurogastroenterología ante CCv4.0 analicen cada una de estas revisiones técnicas, lo cual será publicados en los próximos números del 2022.

El segundo artículo de este número, por FeboRodríguez, se refiere a la dispepsia funcional en niños ${ }^{5}$. La prevalencia global de dispepsia funcional en niños es del $4.5 \%$. Esta alta prevalencia también se manifiesta en Latinoamérica, donde la dispepsia funcional es el TIIC más frecuente asociado con dolor abdominal ${ }^{6}$. De hecho, un estudio llevado a cabo en 3,567 niños de seis ciudades de Colombia, encontró que el $21 \%$ de los niños tenían al menos un TIIC y que el 3\% de todos los niños tenía dispepsia funcional, siendo la forma asociada con molestia posprandial su presentación más frecuente $(2.7 \%)^{6}$. La inclusión de este subgrupo de dispepsia funcional en los criterios de Roma IV en pediatría es la causa del aumento de la frecuencia del diagnóstico de dispepsia funcional en niños que se ha visto entre las versiones de Roma III a IV7.

Este diagnóstico tiene importantes implicaciones en términos de calidad de vida y de trastornos del sueño en los niños que padecen este trastorno ${ }^{8}$. Además, la correcta evaluación y manejo de dispepsia funcional en pediatría también tiene un importante impacto económi$\mathrm{CO}^{9}$. Un estudio mostró que los costos asociados con dispepsia funcional son enormes en EE.UU., con una estimación de $\$ 5.79$ billones de dólares. En el artículo de este número, Febo-Rodríguez nos acerca al entendimiento del enigma de la fisiopatología de la dispepsia funcional y su correcto manejo ${ }^{5,10}$. Esto es de particular importancia para evitar el impacto negativo de esta patología en la vida del niño y su familia, así como desde el punto de vista económico, ya 
que los costos de su manejo con pobres resultados son significativamente mayores ${ }^{9}$. Nuevos estudios deberán proveer evidencia sobre la optimización de su investigación y manejo, así como sobre las diferentes estrategias a llevarse a cabo en las diversas regiones del mundo y en particular en Latinoamérica ${ }^{11}$.

Seguidamente, García-Zermeño y Remes-Troche, de la Universidad Veracruzana, revisan la utilidad de la suplementación con fibra en TIIC, especialmente en estreñimiento crónico y en $\mathrm{SII}^{12}$. Es interesante que el concepto de que la fibra acelera el tránsito intestinal y mejora el volumen de las heces siga vigente. Sin embargo, el principal limitante para el uso de la fibra son los efectos secundarios, como por ejemplo la distensión abdominal e incluso el empeoramiento del dolor abdominal, en especial en SII. No se debe olvidar que dosis altas, por ejemplo, mayores de $30 \mathrm{~g}$ de psyllium al día, como bien lo mencionan los autores, pueden retrasar el tránsito de gas y empeorar síntomas relacionados, cosa que comúnmente vemos en la clínica y que debe ser advertido a los pacientes. Otro aspecto que destacar que se incluye en esta revisión es el efecto de la fibra sobre la modulación de la microbiota, la disminución de la inflamación de bajo grado y de la permeabilidad intestinal $^{13,14}$. La revisión también dedica un espacio a las fuentes de obtención de fibra dietética, incluyendo frutas como la tuna, el plátano, el kiwi, el nopal y el agave, y si bien las evidencias son aún limitadas, pueden ser recomendaciones para la práctica clínica diaria ${ }^{12}$. Así mismo, se describen las propiedades fisicoquímicas de la fibra como la viscosidad, fermentabilidad y solubilidad en agua, necesarias para lograr efectos fisiológicos adecuados ${ }^{15}$. Finalmente, también se ilustra el efecto de la fibra en intestino delgado y colon. En el primero, produciendo incremento en la retención de agua y disminución de la reabsorción de sales biliares. En contraste, en el colon, la fibra produce una mayor retención de agua que aumenta el volumen de las heces, estimulando la motilidad colónica. Lo anterior, sin dejar de lado la relación con la microbiota, estimulando el crecimiento de comensales benéficos productores de ácidos grasos de cadena corta como el butirato, necesario para mantener la inmunidad y permeabilidad de la mucosa, entre otros ${ }^{16,17}$.

Por otra parte, los TIIC presentan una fisiopatología multifactorial en la cual hay factores genéticos que predisponen aspectos fisiopatológicos que a su vez se relacionan con factores ambientales, por lo cual se consideran trastornos «complejos» ${ }^{18,19}$. Pero además, siendo trastornos de difícil tratamiento y sin la presencia de tratamientos universalmente efectivos, también los factores farmacogenéticos tienen una importancia en la variabilidad en la respuesta a los tratamientos en subgrupos de pacientes ${ }^{20,21}$. Tal es el caso del transportador de serotonina, cuya expresión está determinada genéticamente y a mayor expresión hay menor serotonina y por lo tanto mayor respuesta a medicamentos bloqueadores de este receptor $^{22,23}$. Con base en lo anterior, en los últimos años ha habido una explosión de estudios de investigación sobre la relación de dichos factores genéticos y farmacogenéticos en los TIIC. Es por ello que los neurogastroenterólogos debemos estar familiarizados con estos conceptos, razón por lo cual en este número de NeuroGastroLATAM Reviews, Lozano-Hernández y Gutiérrez-Aguilar, dos expertos en la materia, publican la revisión Genética y genómica en el síndrome de intestino irritable: una introducción 
para el neurogastroenterólogo ${ }^{24}$. Este artículo tiene por objeto revisar de manera didáctica los fundamentos de la genética y genómica que requerimos para entender los avances de la neurogastroenterología en el campo de los TIIC. Además de definir dichos fundamentos, utilizan al SII como ejemplo de un trastorno poligénico, para revisar las diversas estrategias que se utilizan para descubrir las variantes genéticas relacionados con el desarrollo de este trastorno o que confieren susceptibilidad para este; las diversas cohortes que se utilizan como estudios de agregación familiar, casos y controles, y estudios poblacionales; los enfoques utilizados como genes candidatos cuya función se conoce antes de la investigación, hasta los estudios de asociación de genoma completo, en los cuales los polimorfismos se seleccionan al azar sin conocer su función pero cubriendo el genoma completo ${ }^{24}$. Este tema, si bien es de suma importancia, no es fácil de comprender, pero estamos seguros que la presente revisión será clave como artículo de consulta y educación en la materia.

De esta forma concluye el año 2021 en NeuroGastroLATAM Reviews. Un año marcado por un retardo en el progreso de la investigación científica producto de la gran pandemia de nuestra generación, la enfermedad por coronavirus 2019, pero también marcado por la vacunación contra el coronavirus 2 del síndrome respiratorio agudo grave, su agente causal.

\section{BIBLIOGRAFÍA}

1. Yadlapati R, Kahrilas PJ, Fox MR, Bredenoord AJ, Prakash Gyawali C, Roman S, et al. Esophageal motility disorders on high-resolution manometry: Chicago classification version 4.0((c)). Neurogastroenterol Motil. 2021;33:e14058.
2. Yadlapati R, Pandolfino JE, Fox MR, Bredenoord AJ, Kahrilas PJ. What is new in Chicago Classification version 4.0? Neurogastroenterol Motil. 2021;33:e14053.

3. Herbella FAM, del Grande LM, Schlottmann F, Patti MG. Changes in the treatment of primary esophageal motility disorders imposed by the New Classification for Esophageal Motility Disorders on High Resolution Manometry (Chicago Classification 4.0). Adv Ther. 2021;38:2017-26.

4. Achem SR, Vázquez- Elizondo G. Hypercontractile esophagus: A practical review. NeuroGastroLatam Rev. 2021;5:6-15.

5. Febo-Rodríguez L. Pediatric functional dyspepsia: A comprehensive review. NeuroGastroLatam Rev. 2021;5

6. Saps M, Velasco-Benítez CA, Langshaw AH, Ramírez-Hernández CR. Prevalence of functional gastrointestinal disorders in children and adolescents: Comparison between Rome III and Rome IV Criteria. J Pediatr. 2018;199:212-6.

7. Baaleman DF, Di Lorenzo C, Benninga MA, Saps M. The effects of the Rome IV Criteria on pediatric gastrointestinal practice. Curr Gastroenterol Rep. 2020;22:21.

8. Schurman JV, Friesen CA, Dai H, Danda CE, Hyman PE, Cocjin JT. Sleep problems and functional disability in children with functional gastrointestinal disorders: an examination of the potential mediating effects of physical and emotional symptoms. BMC Gastroenterol. 2012;12:142.

9. Mani J, Madani S, Thomas R. Economic impact and prognostic factors of functional dyspepsia in children. J Pediatr Gastroenterol Nutr. 2020;70:e65-e70.

10. Romano C, Valenti S, Cardile S, Benninga MA. Functional dyspepsia: An enigma in a conundrum. J Pediatr Gastroenterol Nutr. 2016;63:579-84.

11. Dainton C, Chu C. Symptom clusters on primary care medical service trips in five regions in Latin America. J Epidemiol Glob Health. 2015;5:259-64.

12. García-Zermeño KR, Remes-Troche JM. Fibra en los trastornos de la interacción intestinocerebro. NeuroGastroLatam Rev. 2021;5.

13. Jha R, Mishra P. Dietary fiber in poultry nutrition and their effects on nutrient utilization, performance, gut health, and on the environment: a review. J Anim Sci Biotechnol. 2021;12:51

14. Shah BR, Li B, Al Sabbah H, Xu W, Mráz J. Effects of prebiotic dietary fibers and probiotics on human health: With special focus on recent advancement in their encapsulated formulations. Trends Food Sci Technol. 2020;102:178-92.

15. Williams BA, Grant LJ, Gidley MJ, Mikkelsen D. Gut fermentation of dietary fibres: Physico-chemistry of plant cell walls and implications for health. Int J Mol Sci. 2017;18:2203.

16. Kim MY, Choi SW. Dietary modulation of gut microbiota for the relief of irritable bowel syndrome. 2021;15:411-30.

17. Akagawa S, Akagawa Y, Nakai Y, Yamagishi M, Yamanouchi S, Kimata T, et al. Fiber-rich barley increases butyric acid-producing bacteria in the human gut. Microbiota. 2021;11:559.

18. Mahurkar-Joshi S, Chang L. Epigenetic mechanisms in irritable bowel syndrome. Front Psychiatry. 2020;11:805.

19. Xiao QY, Fang XC, Li XQ, Fei GJ. Ethnic differences in genetic polymorphism associated with irritable bowel syndrome. World J Gastroenterol. 2020;26:2049-63

20. Camilleri M, Atanasova E, Carlson PJ, Ahmad U, Kim HJ, Viramontes BE, et al. Serotonin-transporter polymorphism pharmacogenetics in diarrhea-predominant irritable bowel syndrome. Gastroenterology. 2002;123:425-32.

21. Wong BS, Camilleri M, Eckert D, Carlson P, Ryks M, Burton D, et al. Randomized pharmacodynamic and pharmacogenetic trial of dronabinol effects on colon transit in irritable bowel syndrome-diarrhea. Neurogastroenterol Motil. 2012;24:358-e169.

22. Kumar S, Ranjan P, Mittal B, Goshal UC. Serotonin transporter gene (SLC6A4) polymorphism in patients with irritable bowel syndrome and healthy controls. J Gastrointestin Liver Dis. 2012;21:31-8.

23. Vahora IS, Tsouklidis N, Kumar R, Soni R, Khan S. How serotonin level fluctuation affects the effectiveness of treatment in irritable bowel syndrome. Cureus. 2020;12:e9871.

24. Lozano-Hernández L, Gutiérrez-Aguilar R. Genetics and genomics in irritable bowel syndrome: A primer for the neurogastroenterologist. NeuroGastroLatam Rev. 2021;5. 